\title{
Chaos suppression through asymmetric coupling
}

\author{
J. Bragard, ${ }^{\text {a) }}$ G. Vidal, and H. Mancini \\ Department of Physics and Applied Math, Universidad de Navarra, Irunlarrea s/n, \\ E-31080 Pamplona, Spain \\ C. Mendoza \\ Institute of Physics, Pontifical Catholic University of Valparaiso, 234-0025 Valparaiso, Chile
}

S. Boccaletti $i^{\text {b) }}$

CNR-Istituto dei Sistemi Complessi, Via Madonna del Piano 10, 50019 Sesto Fiorentino (FI), Italy

(Received 19 June 2007; accepted 20 September 2007; published online 22 October 2007)

\begin{abstract}
We study pairs of identical coupled chaotic oscillators. In particular, we have used Roessler (in the funnel and no funnel regimes), Lorenz, and four-dimensional chaotic Lotka-Volterra models. In all four of these cases, a pair of identical oscillators is asymmetrically coupled. The main result of the numerical simulations is that in all cases, specific values of coupling strength and asymmetry exist that render the two oscillators periodic and synchronized. The values of the coupling strength for which this phenomenon occurs is well below the previously known value for complete synchronization. We have found that this behavior exists for all the chaotic oscillators that we have used in the analysis. We postulate that this behavior is presumably generic to all chaotic oscillators. In order to complete the study, we have tested the robustness of this phenomenon of chaos suppression versus the addition of some Gaussian noise. We found that chaos suppression is robust for the addition of finite noise level. Finally, we propose some extension to this research. () 2007 American Institute of Physics. [DOI: 10.1063/1.2797378]
\end{abstract}

\begin{abstract}
Chaos suppression has been realized in the past by using techniques related to parameter perturbations as well parametric forcings. In the present paper, we introduce a new technique based on couplings. Here the recipe rests on selecting an adequate coupling able to drive a chaotic dynamics towards a regular periodic attractor.
\end{abstract}

\section{INTRODUCTION}

The question that it is addressed throughout this paper, i.e., chaos suppression through coupling, fits in the more general framework of synchronization. Historically, the regain of interest in the study of synchronization of chaotic systems started back from the seminal work of Pecora and Caroll $^{1}$ in 1990. These authors were able to show experimentally that electronic chaotic circuits were amenable to stable synchronization. These authors also forecast the future applications of their findings in the description of neural processes. Other experimental evidence of synchrony in nonlinear electronic circuits were given by del Rio et al. ${ }^{2}$ these authors used both mutual and unidirectional couplings between chaotic circuits. From the theoretical side, a study of the Japanese Fujisaka and Yamada ${ }^{3}$ as early as 1983 describing the synchronization of chaotic coupled maps demonstrated that chaotic dynamics and synchronization are two concepts that can coexist. There is evidence that synchronization is ubiquitous in nature and some examples are to be found from clapping hands, flashing fireflies, and cardiac and

\footnotetext{
${ }^{a)}$ Electronic mail: jbragard@unav.es

b) Also at The Italian Embassy in Tel Aviv, Trade Tower 25 Hamered Street, Tel Aviv, Israel.
}

neural processes just to cite few of them. Therefore, it is not surprising that synchronization concepts have also percolated in the lay audience literature. ${ }^{4}$ An excellent account of the status of synchronization research can be found in the following, more technical, references. ${ }^{5,6}$

The purpose of this work is to show that an adequate coupling between two identical chaotic oscillators may force their dynamics towards regular periodic oscillations. As counterintuitive as it may seem, the two subsystems oscillate at the same frequency but follow a different limit cycle. The latter means that in terms of synchronization theory they are in a generalized synchronization state. Previous studies on chaos suppression, which is of crucial interest in technological contexts, have focused on different techniques. In 1996, Gutierrez et al. have successfully implemented a method of chaos suppression through changes in the system variables. ${ }^{7}$ In 2001, Kiss and Hudson ${ }^{8}$ showed that by applying an external periodic forcing with a special frequency and amplitude, a chaotic chemical oscillator was forced back to a nonchaotic behavior. In 2002, Patidar et al. ${ }^{9}$ numerically demonstrated the suppression of chaos in coupling two Duffing oscillators, one in the chaotic regime and the other in a periodic regime. In 2005, a Lorenz system driven by a parametric forcing of high frequency as well as with a stochastic forcing was shown to exhibit chaos suppression. ${ }^{10}$ More recently, the study of chaos suppression has been considered for a ring network of activator-inhibitor chaotic reactions in order to mimic biochemical reaction pathways. ${ }^{11}$ In this last study, the authors showed that by adding extra random longrange coupling inside the network the system can exhibit chaos suppression and complete synchronization. While re- 
lated to these previous studies, our analysis is different because we only consider connecting two identical chaotic oscillators in an asymmetric fashion. This is related to one early study of some of the present authors. ${ }^{12}$

Before starting with the technical details of our analysis, let us briefly outline the plan of the article. In the next section, we will successively consider the coupling of identical chaotic oscillators formed by a pair of Roessler, a pair of Roessler in the funnel regime, a pair of Lorenz, and finally a pair of Lotka-Volterra model equations. A close look at the Lyapunov exponents of the global system will reveal that particular values for the coupling lead to chaos suppression and as a by-product to generalized synchronization. In the successive section, we will ensure of the robustness of these synchronized states versus the addition of some Gaussian noise. To end up, we will draw some conclusions and plan some future researches.

\section{CASE STUDIES}

Chaotic systems are not easily amenable for analytical calculations. In most cases, numerical simulations are the only way to penetrate into the complexity of the chaotic dynamics. In this paper, we will rely on numerical simulations on different pairs of chaotic oscillators in order to study the phenomenon of chaos suppression induced by coupling. In order to be as general as possible, we will consider four kinds of chaotic systems. For all of them, the procedure will be the same and is summarized in the following protocol: We take a pair of identical chaotic oscillators and couple them through one of their state variables. The coupling is allowed to be asymmetric and the parameter measuring the asymmetry is called $\theta$. The coupling strength is also varied and is called $\epsilon$. After having coupled the oscillators and let some transient time to elapse, the Lyapunov exponents of the coupled system are evaluated. As a result of the coupling, four options are possible: periodic or chaotic; synchronized or desynchronized behaviors. One observes that when the behavior is periodic one always gets a state of generalized synchronization reducing to three the different scenarios.

\section{A. Roessler in the not funnel regime}

Let us start our analysis with the celebrated Roessler model. ${ }^{13}$ This model is one of the simplest dynamical systems that can exhibit chaotic solutions. It has been used extensively in numerical studies dealing with chaos. The model that we will focus on here is composed by two identical coupled Roessler oscillators,

$$
\begin{aligned}
& \dot{x}_{1}=-\omega_{1} y_{1}-z_{1}+\frac{\epsilon}{2}(1+\theta)\left(x_{2}-x_{1}\right), \\
& \dot{y}_{1}=\omega_{1} x_{1}+a y_{1}, \\
& \dot{z}_{1}=f+z_{1}\left(x_{1}-c\right), \\
& \dot{x}_{2}=-\omega_{2} y_{2}-z_{2}+\frac{\epsilon}{2}(1-\theta)\left(x_{1}-x_{2}\right),
\end{aligned}
$$

$$
\begin{aligned}
& \dot{y}_{2}=\omega_{2} x_{2}+a y_{2}, \\
& \dot{z}_{2}=f+z_{2}\left(x_{2}-c\right),
\end{aligned}
$$

where the first set of parameters that we have used is $a$ $=0.15, f=0.2, c=10, \omega_{1}=1$, and $\omega_{2}=1$. For these parameter values, the Roessler system Eqs. (1)-(3), with $\epsilon=0$ is in a chaotic state characterized by a positive, a negative, and a vanishing Lyapunov exponent and the attractor is in the nofunnel regime which means that one can unequivocally define a phase for the state of the oscillator. ${ }^{5}$ In the following, we are interested in characterizing how the coupling affects the difference between the two oscillators. In general, one can define the state of the system I, Eqs. (1)-(3), with the vector $\mathbf{v}_{1}=\left\{x_{1}, y_{1}, z_{1}\right\}$ and similarly the state of the system II, Eqs. (4)-(6) with the vector $\mathbf{v}_{2}=\left\{x_{2}, y_{2}, z_{2}\right\}$. A perfect synchronized state occurs if $\mathbf{v}_{1}(t)=\mathbf{v}_{2}(t)$ for all time after some transient has brought the systems together. This has been called in the field of synchronization as "complete" synchronization. Others weaker synchronized states have also been identified, phase- and in particular antiphase synchronization is the state of synchrony between two weakly connected identical pendulum clocks as observed by C. Hugyens 400 years ago. A modern setup of the Huygens' experiment is described in Ref. 14. Lag and anticipating synchronization may also be observed if the coupling between the two systems allows for some time delay. ${ }^{15,16}$ These latter forms of synchronization are being investigated for their potential technological applications. ${ }^{17}$ Finally, a "generalized" synchronized state exists, where there is no equality of the two state variables but only a functional relation between them, ${ }^{6,18}$

$$
\mathbf{v}_{1}=\mathbf{F}\left(\mathbf{v}_{2}\right) \quad \forall t>t^{*} .
$$

The requirements on the function $\mathbf{F}$ are time independence and strict monotonicity in its domain of definition. These conditions ensure that given the state of system II $\left(\mathbf{v}_{2}\right)$ we can infer the state of system I $\left(\mathbf{v}_{1}\right)$ and vice versa. It is clear that synchronization will be strongly dependent on the coupling parameter $\epsilon$ and the asymmetry of the coupling $\theta$. While synchronization deals basically with the difference between the dynamics of the two subsystems, here we are also interested in the attractor on which the two subsystems get synchronized.

They are many ways to couple two dynamical systems ${ }^{19}$ and the scheme that we have used here enter in the family of the so-called diffusive coupling. Diffusion is not always symmetric, for example, if the diffusion parameter is not constant $^{20}$ or if it exists an asymmetry due to some constraint as, for example, in physiological membranes that selectively diffuse the ions. ${ }^{21}$ The latter justifies our choice of including a parameter that characterize the asymmetry of the coupling $(\theta)$.

The natural thing to start with is to compute the Lyapunov exponents as a function of the coupling parameters $\epsilon$ and $\theta$. The Lyapunov exponents have been used in the past in synchronization studies as it is reported in Refs. 5 and 6. They also have been successfully used as an indicator for defining phase transition. ${ }^{22}$ In this study, it is sufficient to 
compute the two larger Lyapunov exponents in order to determine and characterize the presence of chaotic or periodic states. In order to compute the first two Lyapunov exponents (LE), we have to integrate Eqs. (1)-(6) and two additional copies of a linearized version of the full system (1)-(6). The numerical integrations are performed using a standard Runge-Kutta algorithm of fourth order and the time step is fixed to $d t=10^{-2}{ }^{23}$ The transient time necessary to erase the effect of the initial condition has been fixed to $9 \times 10^{4}$ for Eqs. (1)-(6) and the additional transient time in the tangent space (for the computation of the Lyapunov spectrum) has been set to $5 \times 10^{4}$ in order to get an accurate determination of the Lyapunov exponents. The time spent on the attractor for the computation of the LE is fixed to 65536 in dimensionless time units and the Gram-Schmidt orthogonalization algorithm used to renormalize the Lyapunov vectors is performed every 50 time steps that corresponds to $t=0.5$. This amounts to a number of orthogonalizations equal to 131072 , which is the most costly part in term of CPU time of the present numerical simulations. The error in the evaluation of the LE is further checked by using the formula er. $=\sigma(\mathrm{LE}) / \max (\mathrm{LE})$, where $\sigma$ is the standard deviation of the maximum (positive) LE. One has found that $e r$. is of order of $2 \%$, which is sufficiently small for the purpose of the present analysis.

The main objective of the present analysis is to show that values of $\epsilon$ and $\theta$ that lead to chaos suppression exist. As a by-product, we will show that for these values of the coupling we also have "generalized" synchronized (GS) states for values of $\epsilon$ well below the threshold of complete synchronization (CS). In the first case treated here (i.e., Roessler no-funnel), let us recall that the threshold for CS is equal to $\epsilon=0.2$ and is independent of the asymmetry $\theta$. Figure 1 displays the value of the first two LEs as a function of the two coupling parameters, i.e., the coupling strength $\epsilon$ and the asymmetry in the coupling $\theta$. From the figure, one clearly observes that when the second LE is vanishing (around $\epsilon$ $=0.2$ ) the system enters in the CS state as previously studied in Ref. 24. The novelty of the present study is to allow for two parameters in the coupling (strength and asymmetry); the latest is measured by the $\theta$ parameter. ${ }^{12}$ As it is seen from Fig. 1, one sees an island where the maximum Lyapunov exponent is vanishing. This occurs roughly in the range $(0.06 \leqslant \epsilon \leqslant 0.08$ and symmetrically in the range $-0.2 \leqslant \theta$ $\leqslant 0.2$ ). Due to the coupling between subsystem I [Eqs. (1)-(3)] and subsystem II [Eqs. (4)-(6)] the dynamical regime has lost its chaotic character. This is not surprising per se because the full coupled system lies in a six-dimensional phase space and therefore the variety of dynamical regimes that one encounters is also enlarged. In particular, some islands exist, where the dynamics is no longer chaotic. In the following, we are interested in further characterizing these islands where periodic behavior is observed.

In order to characterize synchronized states one disposes of some "classical" indicators. ${ }^{12}$ The one that is used here is the Pearson coefficient which is the cross-correlation between the subsystem I [Eqs. (1)-(3)] and the subsystem II [Eqs. (4)-(6)]. As the structure of the Roessler attractor is
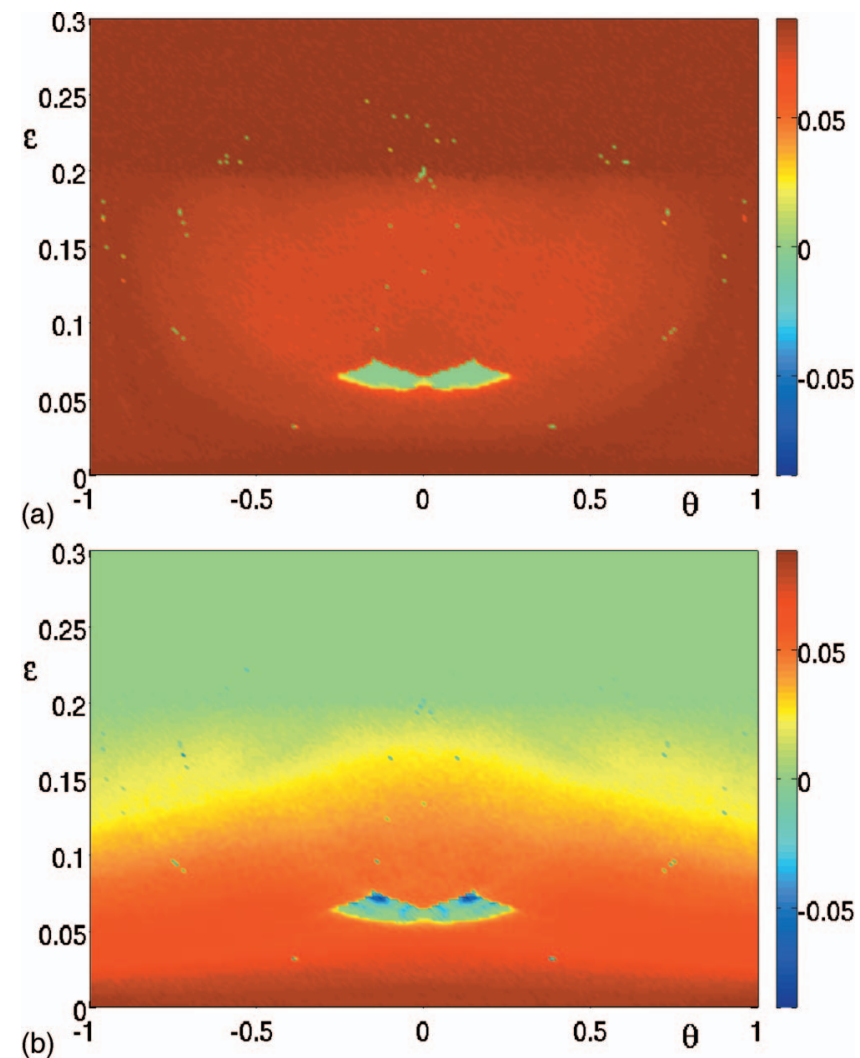

FIG. 1. (Color) The largest (a) and second largest (b) Lyapunov exponent of Eqs. (1)-(6) for the Roessler no-funnel case (see the text for the corresponding parameter values). The horizontal axis indicates the asymmetry of the coupling in the range $-1 \leqslant \theta \leqslant 1$ and the vertical axis is for the coupling strength $0 \leqslant \epsilon \leqslant 0.3$. Note that the same color bar is used for both figures in order to ease the comparison between the two figures.

somewhat particular, it is of interest to separate the cross correlation for the individual variables along the different axes $(x . y . z)$. In doing so one defines

$$
\rho_{x}=\frac{\left\langle\left(x_{1}-\left\langle x_{1}\right\rangle\right)\left(x_{2}-\left\langle x_{2}\right\rangle\right)\right\rangle}{\sigma_{x_{1}} \sigma_{x_{2}}}
$$

which is the cross correlation for the $x$ variable. In the above formula, the brackets $\langle\ldots\rangle$ stand for time average and $\sigma$ stands for standard deviations with respect to the average values. It is well known that when CS occurs all $\rho$ tends to one. However, it is less evident that when GS occurs, the $\rho$ is not necessarily close to one.

Let us evaluate the Pearson coefficient in the first case (Roessler no-funnel).

Figure 2 shows the results of the calculation of the Pearson coefficients. Let us explain in detail how these figures have been obtained. For each couple $(\epsilon, \theta)$, the same initial conditions (each state variables taken randomly in the interval $[0,1]$ ) and the same conditions for transient times and evaluation time for the calculation of the time average are used. Let us mention that some further checks have been done to ensure that changes in the initial conditions do not affect any of the displayed results. From Fig. 2, it is apparent that CS occurs for a value of $\epsilon \approx 0.2$ and that is independent of $\theta$. Also the $\rho_{z}$ tends to one with increasing $\epsilon$ much slower than $\rho_{x}$. This is due to the particular geometry of the Roessler 

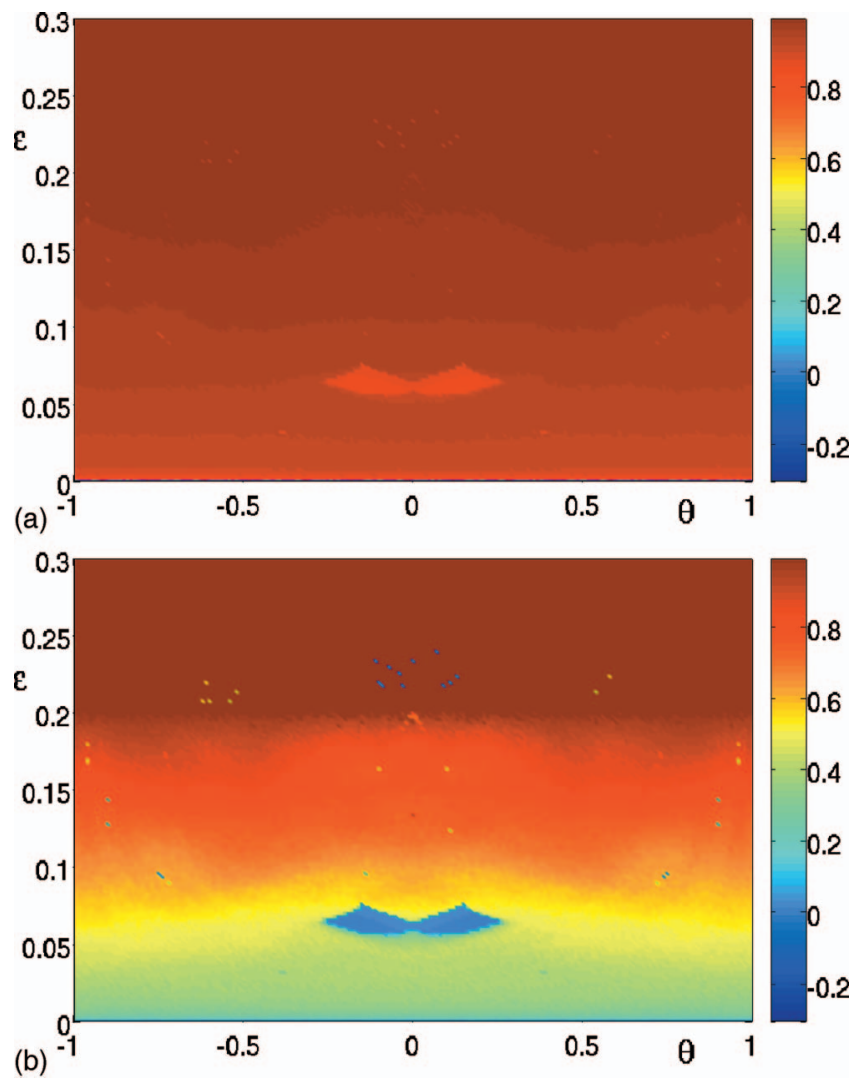

FIG. 2. (Color) Pearson coefficients: at the top (a) $\rho_{x}$ and at the bottom (b) $\rho_{z}$ for the Roessler (no-funnel); see the text for the corresponding parameter values. The horizontal axis is for the asymmetry $-1 \leqslant \theta \leqslant 1$ and the vertical axis is for the coupling strength $0 \leqslant \epsilon \leqslant 0.3$.

attractor. The island that was identified in Fig. 1 is also visible in Fig. 2. However, here the island corresponds to values of the Pearson coefficient lower than the value in the neighborhood. This would lead to the wrong impression that there is a lack of synchronization in this region. In fact, the Pearson indicator is a good indicator for CS but it is a poor indicator for weaker synchronized states. Indeed, one must be reminded that the Pearson indicator is a perfect tool to prove linear correlation between two signals. If we are facing GS, the relation between the two signals is in general not linear and therefore this linear indicator may miss the identification of GS states.

\section{Identification of GS states}

In this paragraph, we show that the region where the maximum LE vanishes corresponds to GS states. In order to identify GS, Abarbanel and co-workers have developed an involved technique called mutual false nearest neighbor. ${ }^{18,25}$ In the present case, it is not necessary to use this method, which is computationally demanding, because the attractors in each of the two subsystems are not chaotic. It is straightforward to find a function that relates $\mathbf{v}_{1}$ and $\mathbf{v}_{2}$ if both states are periodic.

It is seen from Fig. 3(a) that the dynamics loses its chaotic character if the coupling is set to $\epsilon=0.07$ and $\theta=0.15$ which are values taken in the island identified in Fig. 1. In addition, it is shown in Fig. 3(b), that the projection in the plane $\left(x_{1}, x_{2}\right)$ is a curve rather than a cloud of points as it

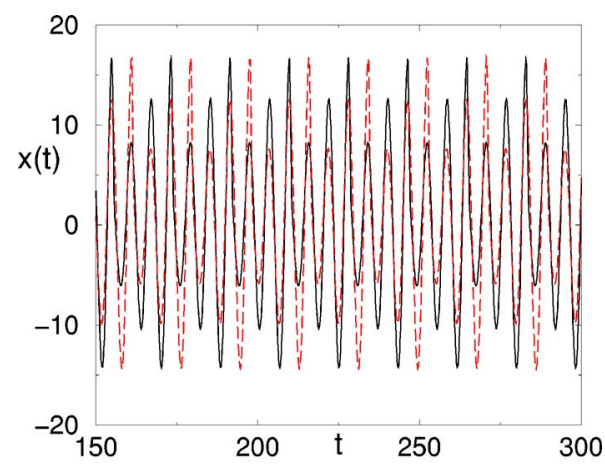

(a)

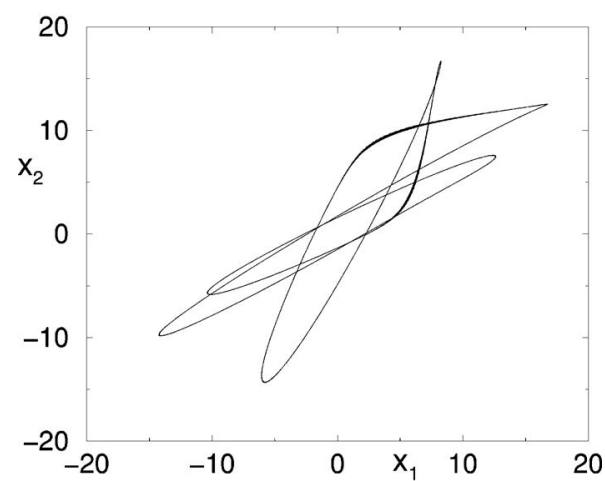

(b)

FIG. 3. (Color online) (a) time evolution of $x_{1}(t)$ solid line and $x_{2}(t)$ dashed line. (b) the projection of the dynamics on the plane $\left(x_{1}, x_{2}\right)$, which is reminiscent of a Lissajous figure. Coupling values are $\epsilon=0.07$ and $\theta=0.15$ (the others parameters are for the Roessler no-funnel, see the text for parameter values).

would be if the dynamics were chaotic. When the system is in a CS state, the curve in Fig. 3(b) is a straight line (the first bisector). Here below, we show that the system is in a GS state. Before evaluating the function relating $\mathbf{v}_{\mathbf{1}}$ and $\mathbf{v}_{\mathbf{2}}$, let us display the attractor for these particular parameter values $(\epsilon$ $=0.07$ and $\theta=0.15$ ).

Figure 4 shows that the selected coupling values $(\epsilon$ $=0.07$ and $\theta=0.15$ ) are able to restore a regular (not chaotic) dynamics. It is important to note that the value of the coupling strength is well below (three times lower than) the threshold of CS $(\epsilon \approx 0.2)$. Surprisingly and left unnoticed by

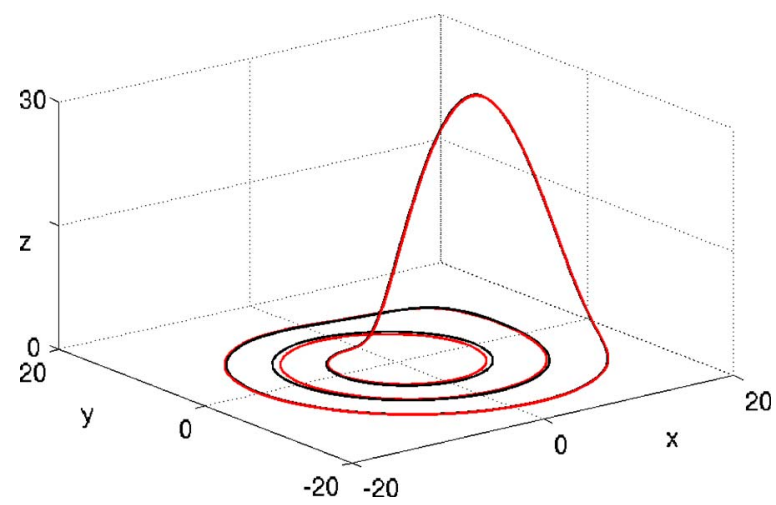

FIG. 4. (Color online) Plot of the two attractors: $\mathbf{v}_{\mathbf{1}}$ and $\mathbf{v}_{\mathbf{2}}$ in the case of $\epsilon=0.07$ and $\theta=0.15$. The two curves nearly coincide and chaos has disappeared due to the particular coupling between the two chaotic oscillators. 


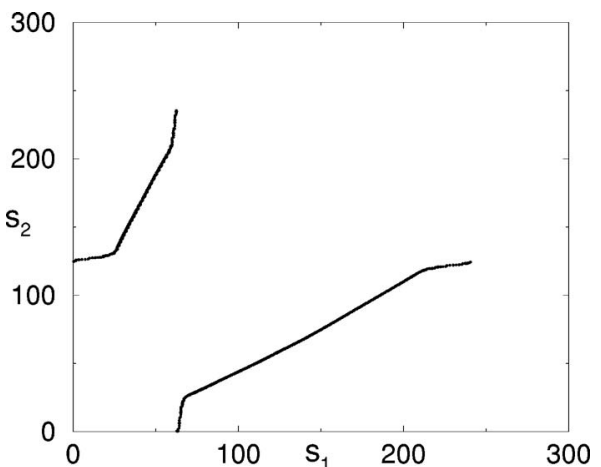

FIG. 5. Plot of the arc length along the two attractors: $s_{1}$ and $s_{2}$ are taken from an arbitrary (but common) origin. $\epsilon=0.07$ and $\theta=0.15$.

previous studies, for such a low value of the coupling strength, the system lies in a state of GS. As it is seen from Fig. 4 the system is looping in the $(x, y)$ plane and it is not directly related to the state $\mathbf{v}_{\mathbf{1}}$ and $\mathbf{v}_{2}$. The easiest way to proceed is to define two sets of curvilinear coordinates along the parametric curves $\mathbf{v}_{\mathbf{1}}(t)$ and $\mathbf{v}_{\mathbf{2}}(t)$ that measure the arc length along the attractor I and attractor II. The origin of these coordinates is arbitrarily chosen to be where $z(t)$ is maximum. The arc lengths are denoted by $s_{1}(t)$ and $s_{2}(t)$, respectively. After each period (cycle) on the attractor, we reset the arc length to zero. If a GS state exists, given any location in subsystem I, one is able to find unequivocally the corresponding location on the attractor II, and this will happen for any time.

Figure 5 reveals that for $\epsilon=0.07$ and $\theta=0.15$, the coupling of the two identical Roessler attractors reduce to two periodic attractors that are slightly different. If we know the state of one of the two subsystems $\mathbf{v}_{\mathbf{1}}$ or $\mathbf{v}_{\mathbf{2}}$, we can predict the other. Indeed, the nonlinear function relating $s_{1}$ and $s_{2}$ is monotonously increasing and it is time independent. Note that the discontinuity in the function is not a problem and it is due to the specific choice of the origin of coordinates. If one repeats the same procedure for other coupling values $(\epsilon$, $\theta$ ) situated in the island where the maximum Lyapunov exponent vanishes, one finds that a generalized synchronized state always exists. If one compares the results of the present work with the one of Rulkov et al., ${ }^{25}$ one sees that here GS comes from the particular coupling between two identical Roessler attractors. On the contrary, in Rulkov analysis, there was already a CS state that is "artificially" transformed into GS by a nonlinear change of variables. The objective of Rulkov et al. was to prove that their method of detecting GS is relevant. They actually demonstrated that the false mutual nearest neighbor method is indeed an excellent method to detect GS states between two (or more) chaotic oscillators.

\section{B. Roessler in the funnel regime}

The first question that immediately arises after observing these GS states is the following: Is this phenomenon of suppression of chaos by asymmetric coupling generic? To test this hypothesis one has to carefully check several issues. First of all, one has to ascertain that different random initial conditions do not modify the conclusions. This has been

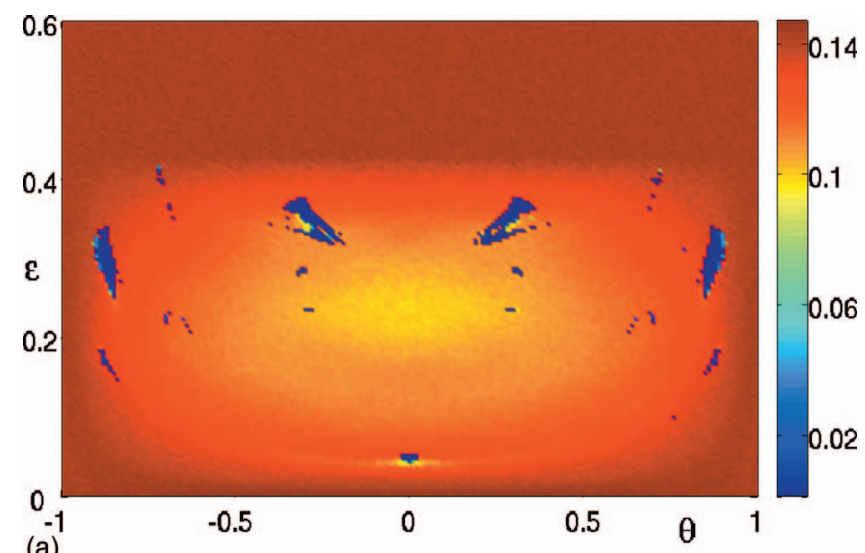

(a)

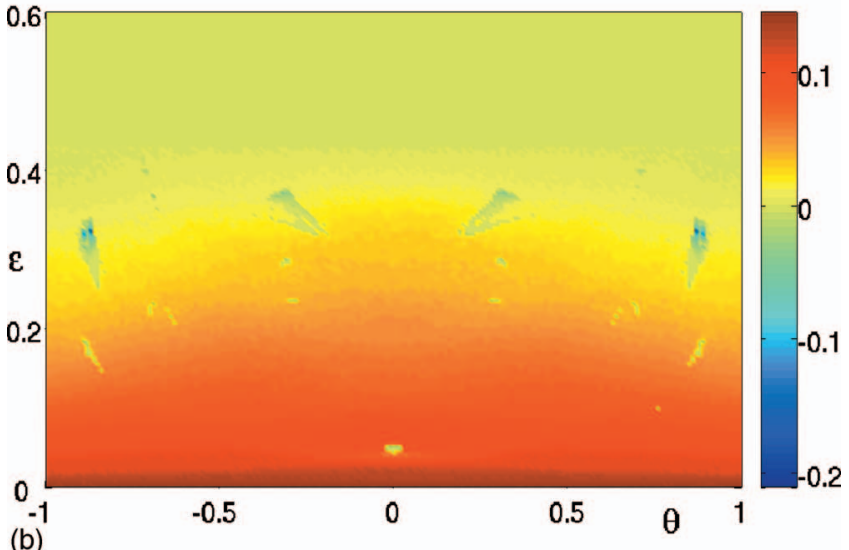

FIG. 6. (Color) The largest (a) and second largest (b) Lyapunov exponent of Eqs. (1)-(6) for the funnel case (see the text for the corresponding parameter values). The horizontal axis is for the asymmetry $-1 \leqslant \theta \leqslant 1$ and the vertical axis is for the coupling strength $0 \leqslant \epsilon \leqslant 0.6$. Note that the epsilon range has been doubled with respect to the no-funnel case.

checked successfully. Furthermore, we have to check that this phenomenon is not specific to a particular choice of attractor. It may well be that one is observing a GS phenomenon just by chance. This phenomenon will be called generic if it happens for different values of the parameters and also for a different class of chaotic attractors (like Lorenz attractor, Lotka-Volterra model, etc.).

In this section, the first test that we have performed is to modify the parameter values of the Roessler attractor. In particular by fixing the values of the parameters in Eqs. (1)-(6) to $a=0.343, f=1.82, c=9.75, \omega_{1}=1$, and $\omega_{2}=1$, the attractor transforms into a funnel attractor ${ }^{26}$ which is topologically very different from the no-funnel attractor (regular case) that we have analyzed in the previous section.

If the attractor is very different one may suspect that its response to coupling will also be different. In this section, we have redone the same analysis as in the previous section but for the funnel attractor. Figure 6 shows that the presence of islands where the maximum LE vanish is again observed. In this case, the transition to CS is obtained for $\epsilon \approx 0.4$ and it is still independent of the asymmetry of the coupling $\theta$. In Fig. 6 , one identifies a small island of regular behavior for $\epsilon$ $\approx 0.05$ and $\theta=0$. This indicates that regular periodic behavior can be obtained even in the absence of asymmetry in the coupling. Asymmetrical coupling surely enhances the number of islands where regular behavior is observed but it is not 


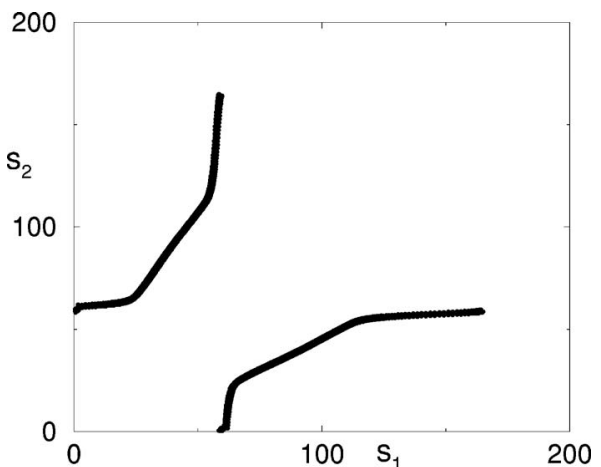

FIG. 7. Plot of the arc length relation in the plane $\left(s_{1}, s_{2}\right)$ for the funnel case and $\epsilon=0.05, \theta=0$.

a necessary condition for its occurrence as we see in Fig. 6. A close look at the dynamical system when $\epsilon \approx 0.05$ and $\theta=0$ indeed shows that the dynamics of subsystems I and II is periodic and again the system is in a GS state as it is proven in Fig. 7.

It is important to mention that regular (nonchaotic) behavior does not imply at all that the system will automatically lie in a GS state. Inside each island, one has to check if there is a function relating the states of the two subsystems. One has to be able to predict the state of $\mathbf{v}_{\mathbf{1}}$ from $\mathbf{v}_{\mathbf{2}}$ and vice versa. Here we have verified that in each island we have GS states. This is presumably due to the fact that if two identical systems (initially chaotic) are coupled and fall into a periodic attractor, they very likely be synchronized.

\section{Lorenz attractors}

In this section, we repeat the procedure of the previous two sections but this time working with a pair of coupled Lorenz attractors, ${ }^{27}$

$$
\begin{aligned}
& x_{1}=a\left(y_{1}-x_{1}\right)+\frac{\epsilon}{2}(1+\theta)\left(x_{2}-x_{1}\right), \\
& y_{1}=x_{1}\left(r-z_{1}\right)-y_{1}, \\
& z_{1}=x_{1} y_{1}-b z_{1}, \\
& x_{2}=a\left(y_{2}-x_{2}\right)+\frac{\epsilon}{2}(1-\theta)\left(x_{1}-x_{2}\right), \\
& y_{2}=x_{2}\left(r-z_{2}\right)-y_{2}, \\
& z_{2}=x_{2} y_{2}-b z_{2},
\end{aligned}
$$

where the chosen parameter values are $a=10, r=28$, and $b=8 / 3$. For these values the Lorenz system is chaotic and the attractor displays its well-known "butterfly" shape (see Fig. 8).

Again, with the Lorenz attractor, one finds areas in the $(\epsilon, \theta)$ plane where the coupling eliminates the chaotic character of the attractor and imposes a periodic regime as it is exemplified in Fig. 7(b) for parameter values $(\epsilon=3.7$ and $\theta$ $=0.2$ ). Figure 7(b) exemplifies that the two subsystems do not fall on the same attractor. However, we have checked
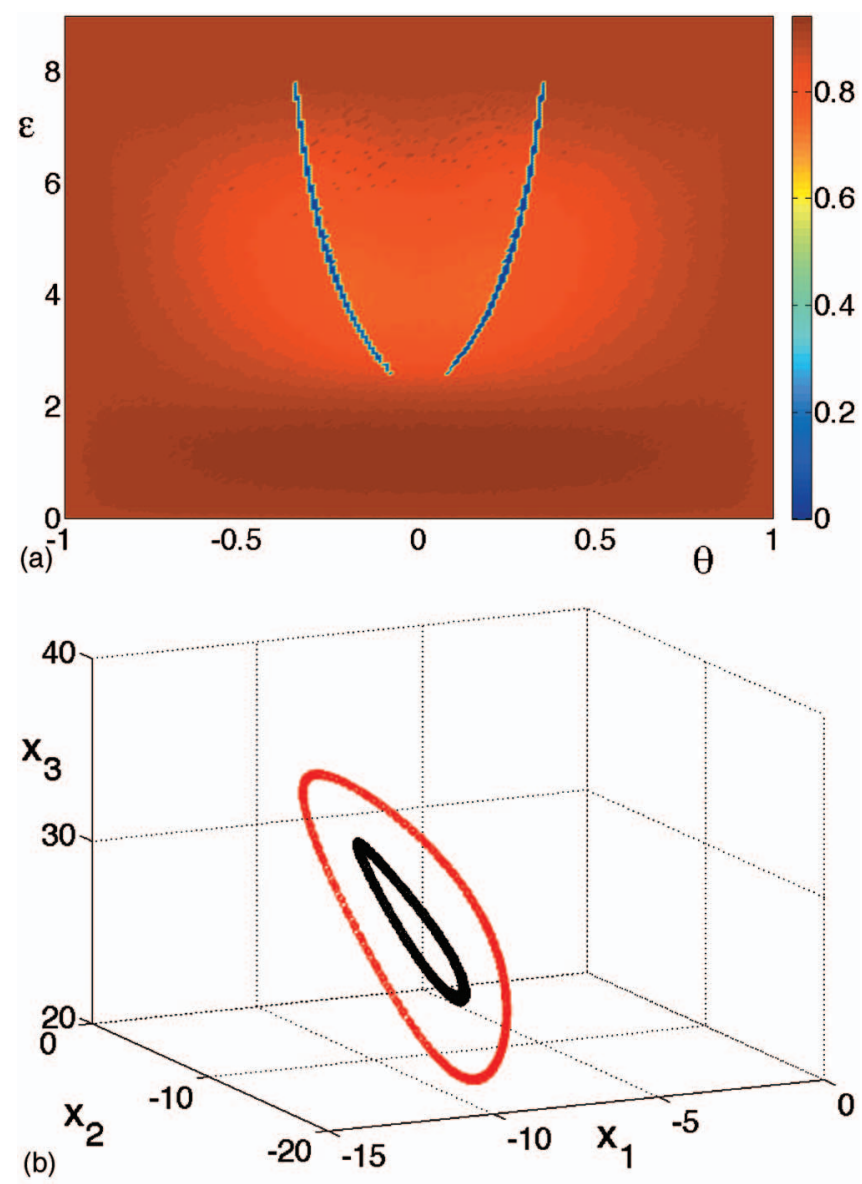

FIG. 8. (Color) The largest (a) Lyapunov exponent for the Lorenz system; blue regions represent the area where the maximum Lyapunov exponent is vanishing (b). Projections of the attractors $\mathbf{v}_{\mathbf{1}}$ (black curve) and $\mathbf{v}_{\mathbf{2}}$ (red curve) when $(\epsilon=3.7$ and $\theta=0.2)$, showing that the system is no longer chaotic.

that the frequencies are the same which is also a different way to ensure for GS between periodic attractors.

Even if the Lorenz attractor has a maximum Lyapunov exponent one order of magnitude larger than the Roessler attractor, the same behavior is encountered. Obviously, the value for which one gets complete synchronization is much higher than in the Roessler case and is close to $\epsilon=8$ and again independent of $\theta$.

\section{Lotka-Volterra model}

To end up with case studies, let us consider a model coming from Ecology which is the four-dimensional LotkaVolterra for competitive species, ${ }^{28,29}$

$$
\begin{aligned}
& \dot{x}_{i}^{(1)}=r_{i} x_{i}^{(1)}\left(1-\sum_{k=1}^{4} \alpha_{i k} x_{k}^{(1)}\right)+\delta_{i, 1} \frac{\epsilon}{2}(1+\theta)\left(x_{1}^{(2)}-x_{1}^{(1)}\right), \\
& \dot{x}_{i}^{(2)}=r_{i} x_{i}^{(2)}\left(1-\sum_{k=1}^{4} \alpha_{i k} x_{k}^{(2)}\right)+\delta_{i, 1} \frac{\epsilon}{2}(1-\theta)\left(x_{1}^{(1)}-x_{1}^{(2)}\right),
\end{aligned}
$$

where in Eqs. (15), $i=1, \ldots, 4$ is an equation index and the superscript indicates subsystem I or subsystem II. The coupling, as usual, is realized only over the first variable and 


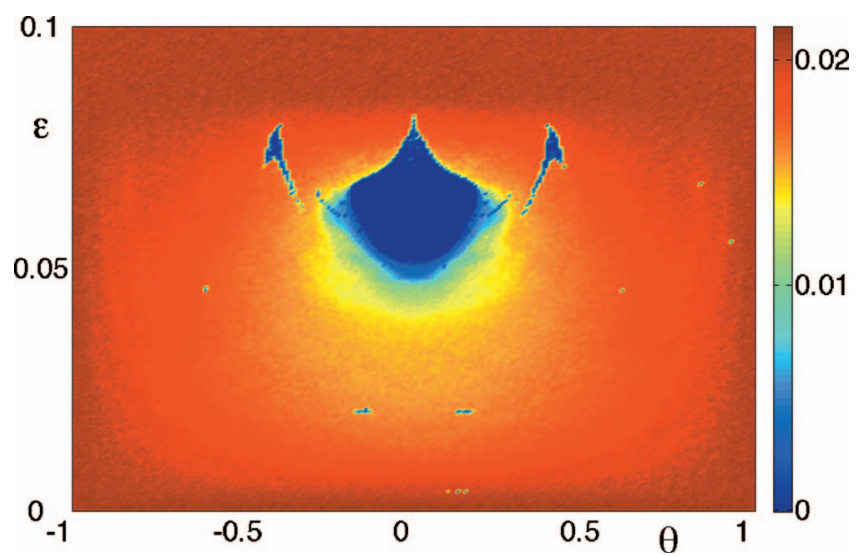

FIG. 9. (Color) The largest Lyapunov exponent for the Lotka-Volterra system [Eqs. (15)]; blue regions represent the area where the maximum Lyapunov exponent is vanishing (see text for parameter values).

therefore to have a consistent notation, we have used the usual Kronecker symbol $\delta_{i, 1}$ in order to condense the notation. The parameters are identical for the two system and are given by $\mathbf{r}^{T}=[1 ; 0.72 ; 1.53,1.27]$ and the interspecies matrix is given by

$$
\boldsymbol{\alpha}=\left[\begin{array}{cccc}
1 & 1.09 & 1.52 & 0 \\
0 & 1 & 0.44 & 1.36 \\
2.33 & 0 & 1 & 0.47 \\
1.21 & 0.51 & 0.35 & 1
\end{array}\right]
$$

for the parameter values given in the above matrix, the competitive species model is in the chaotic range. The populations of the four species in the two subsystems fluctuate in a chaotic manner.

As it appears clearly from Fig. 9, here again the coupling between the two groups of population species also lead to chaos suppression for some values of the coupling $\epsilon$ and $\theta$. All these numerical explorations give confidence on the generality of the observed phenomenon.

\section{DISCUSSIONS ABOUT ROBUSTNESS}

Finally we are interested in testing if this phenomenon of chaos suppression is robust. It is well known that synchronized states are highly vulnerable to noise added to the synchronizing signal. ${ }^{30}$ In the following, some noise is added to the coupling term in order to test for the robustness. The noise is white Gaussian noise with some strength (characterized by its variance). By the addition of a noisy term in each of the coupling terms, the equations become a set of stochastic differential equations. ${ }^{31}$ Some careful treatment is in order in the numerical integration of these stochastic equations. The addition of some noise permit us to test if we are not facing mere numerical artifacts. Two white Gaussian noises, $\eta_{1}$ and $\eta_{2}$, are added to Eqs. (1) and (4), respectively. The strength of the noise is measured by the standard deviation of the distribution, we have $\left\langle\eta_{1}^{2}\right\rangle=\left\langle\eta_{2}^{2}\right\rangle=\sigma_{r}^{2}$. Obviously the noise is chosen to have a zero mean, $\left\langle\eta_{1}\right\rangle=\left\langle\eta_{2}\right\rangle=0$.

Figure 10(a) shows that the addition of a noise with $\sigma_{r}$ $=0.01$ is not able to completely destroy the phenomenon of chaos suppression that is observed for $\epsilon \approx 0.05$ and $\theta=0$ in

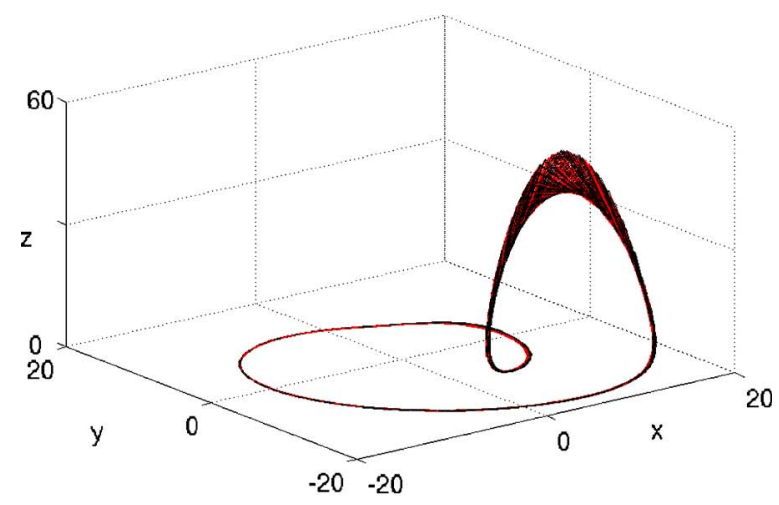

(a)

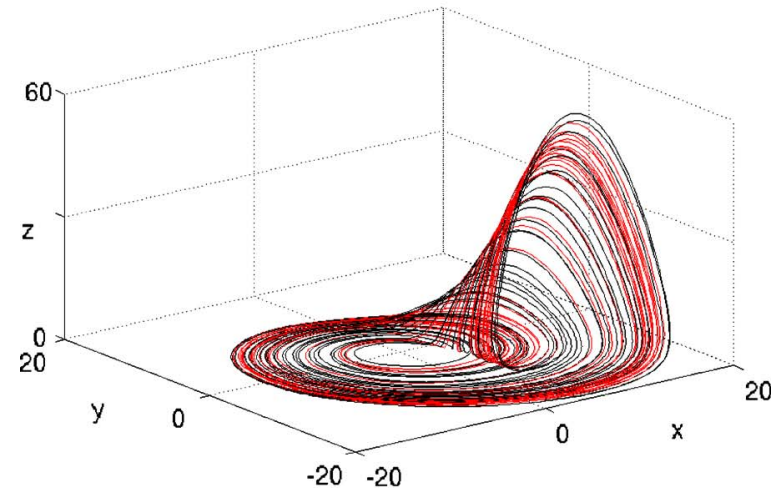

(b)

FIG. 10. (Color online) The influence of noise on the GS states: (a) white Gaussian noises with $\sigma_{r}=0.01$ are added to Eqs. (1) and (4) of the Roessler attractor in the funnel region [case study (b)], the chaos suppression is still present. (b) $\sigma_{r}=1$ and the attractors are back in the chaotic region. Parameter values are $\epsilon=0.05$ and $\theta=0$.

the Roessler (funnel regime) in the absence of noise. However, due to the noisy term, the attractor is logically smeared out, especially noticeable in the region of large $z$ where the expansion of the attractor is larger. On the contrary, the addition of a larger noise [see Fig. 10(b)] restores the two subsystems into chaotic dynamics.

A further check is done by increasing the added noise from very small values to larger values. The objective is to capture the transition (noise strength) for which the chaotic behavior is restored. Figure 11 displays the maximum Lyapunov exponent as a function of the increasing noise level. It is clear from the figure that above a critical noise strength that depends on the particular dynamical system chaos suppression by coupling is no longer present.

\section{CONCLUSIONS}

We have found by extensive numerical simulations that a two parameter coupling $(\epsilon, \theta)$ enlarges the number of situations that one may encounter in coupling a pair of identical chaotic oscillators. For some specific values of the coupling, we have identified four classes of chaotic oscillators to get chaos suppression. This phenomenon seems to have been unnoticed so far, at least between two identical chaotic oscillators. In the framework of synchronization, this chaos suppression is associated with GS states. The regions where GS appears are spread out in the $(\epsilon, \theta)$ plane and are character- 


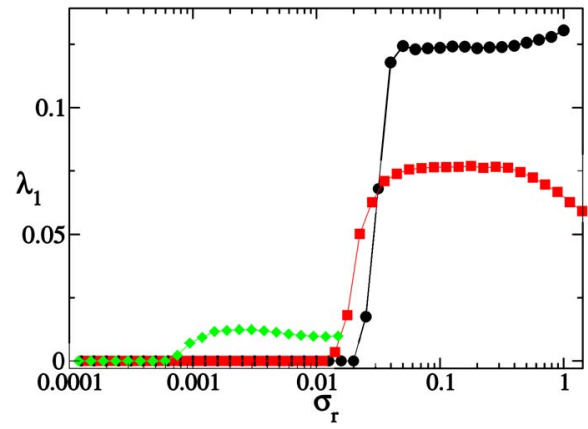

FIG. 11. (Color online) Influence of some added white Gaussian noise on the phenomenon of chaos suppression. The curve marked with circles is for the Roessler oscillators (funnel) with $\epsilon=0.05$ and $\theta=0$. The curve marked with squares is for the Roessler oscillators (nonfunnel regime) with $\epsilon$ $=0.07$ and $\theta=0.15$. The curve marked with diamonds is for the LotkaVolterra model with $\epsilon=0.06$ and $\theta=0$. Note that we have used the logarithmic scale for the noise strength.

istic of each dynamical system. They are like their GS fingerprints. Ideally one would like to get some analytical predictions about the localization and the extent of those regions where chaos suppression occurs. This important task is left for further studies. In this paper, we have shown that chaos suppression is a generic behavior and that is also robust versus the addition of noise. This indicates that the basin of attraction of these states is large enough to attract the trajectories in them. We finally want to conjecture that natural and biological systems may presumably use these specific couplings in order to facilitate synchronization (communication) between their different subunits.

\section{ACKNOWLEDGMENTS}

The authors acknowledge fruitful discussions with B. Echebarria, D. Maza, and E. Alvarez-Lacalle. J.B. acknowledges financial support from MEC Project No. (Spain) FIS2005-06912-C02 (DINCARD). C. Mendoza acknowledges financial support through PBCT Project No. PSD-06. S.B. acknowledges the Yeshaya Horowitz Association through the Center for Complexity Science.
${ }^{1}$ L. Pecora and T. Caroll, Phys. Rev. Lett. 64, 821 (1990).

${ }^{2}$ E. del Rio, M. G. Velarde, A. Rodriguez-Lozano, N. Rulkov, and A. R. Volkovskii, Int. J. Bifurcation Chaos Appl. Sci. Eng. 4, 1003 (1994).

${ }^{3}$ H. Fujisaka and T. Yamada, Prog. Theor. Phys. 69, 32 (1983).

${ }^{4}$ S. Strogatz, Sync: The Emerging Science of Spontaneous Order (Hyperion, New York, 2003).

${ }^{5}$ A. Pikovsky, M. Rosenblum, and J. Kurths, Synchronization-A Unified Approach to Nonlinear Science (Cambridge University Press, Cambridge, 2001).

${ }^{6}$ S. Boccaletti, J. Kurths, G. Osipov, D. L. Valladares, and C. S. Zhou, Phys. Rep. 366, 1 (2002).

${ }^{7}$ J. Gutierrez, A. Iglesias, J. Guemez, and M. Matias, Prog. Theor. Phys. 7, 1305 (1996).

${ }^{8}$ I. Kiss and J. Hudson, Phys. Rev. E 64, 046215 (2001).

${ }^{9}$ V. Patidar, N. K. Pareek, and K. K. Sud, Phys. Lett. A 304, 121 (2002).

${ }^{10}$ C.-U. Choe, K. Hohne, H. Benner, and Y. Kivshar, Phys. Rev. E 72, 036206 (2005).

${ }^{11}$ S. Rajesh, Su. Sinha, and So. Sinha, Phys. Rev. E 75, 011906 (2007).

${ }^{12}$ J. Bragard, S. Boccaletti, and H. Mancini, Phys. Rev. Lett. 91, 064103 (2003).

${ }^{13}$ O. Roessler, Appl. Phys. Lett. 35A, 397 (1972).

${ }^{14}$ M. Bennett, M. F. Schatz, H. Rockwood, and K. Wiesenfeld, Proc. R. Soc. London, Ser. A 458, 563 (2002).

${ }^{15}$ H. U. Voss, Phys. Rev. E 61, 5115 (2000).

${ }^{16}$ H. U. Voss, Phys. Rev. Lett. 87, 014102 (2001)

${ }^{17}$ N. J. Corron, J. N. Blakely, and S. D. Pettel, Chaos 15, 023110 (2005).

${ }^{18} \mathrm{H}$. Abarbanel, Analysis of Observed Chaotic Data (Springer, New York, 1995).

${ }^{19}$ M. Matias and J. Guemez, Phys. Rev. Lett. 72, 1455 (1994).

${ }^{20} \mathrm{~J}$. Crank, The Mathematics of Diffusion, 2nd ed. (Oxford University Press, Oxford, 1980)

${ }^{21}$ P. Nelson, Biological Physics: Energy, Information, Life (Freeman, New York, 2003).

${ }^{22}$ J. Barre and T. Dauxois, Europhys. Lett. 55, 164 (2001).

${ }^{23}$ W. Press, S. Teukolsky, W. Vetterling, and B. Flannery, Numerical Recipes in Fortran 90: The Art of Scientific Computing (Cambridge University Press, Cambridge, 1992).

${ }^{24}$ M. Rosenblum, A. Pikovsky, and J. Kurths, Phys. Rev. Lett. 78, 4193 (1997).

${ }^{25}$ N. Rulkov, M. Sushchik, L. Tsimring, and H. Abarbanel, Phys. Rev. E 51, 980 (1995).

${ }^{26}$ On the web page http://bill.srnr.arizona.edu/demos/rossler/rossler2.html is shown the structure of the Roessler attractor in the funnel and no-funnel cases.

${ }^{27}$ E. Lorenz, J. Atmos. Sci. 20, 130 (1963).

${ }^{28}$ S. Smale, J. Math. Biol. 3, 5 (1976).

${ }^{29}$ J. Vano, J. Wildenberg, M. Anderson, J. Noel, and J. Sprott, Nonlinearity 19, 2391 (2006).

${ }^{30}$ T. Caroll, Chaos 12, 275 (2002).

${ }^{31}$ P. Kloeden and E. Platen, Numerical Solution of Stochastic Differential Equations (Springer-Verlag, Berlin, 1992). 